\title{
Oficinas educativas virtuais de nutrição para portadores de sofrimento mental no contexto da COVID-19: um relato de experiência
}

\author{
Virtual educational nutrition workshops for people with mental suffering in the context of COVID-
} 19: an experience report

Talleres de nutrición educativa virtual para personas con sufrimiento mental en el contexto del COVID-19: informe de experiencia

\section{Resumo}

Com o contexto pandêmico, devido à COVID-19, o distanciamento social se tornou imprescindível como forma de prevenção, fazendo necessário que as ações de pesquisa e extensão se reinventassem. $\mathrm{O}$ trabalho em questão, tem como objetivo relatar a experiência do projeto Educação Nutricional para Portadores de Sofrimento Mental atuando em um Centro de Convivência e Cultura de Belo Horizonte-MG, durante o ano de 2020, no contexto de pandemia da COVID-19. Trata-se de um relato descritivo de experiência. Inicialmente, a abordagem se deu por meio de um material digital em formato de flyers enviados pelo aplicativo WhatsApp aos usuários do Centro de Convivência. Com a necessidade de maior aproximação com os usuários foram produzidos vídeos educativos animados também enviados pelo aplicativo WhatsApp. Durante os meses de março de 2020 a março de 2021, foram produzidos oito flyers com temáticas voltadas à prevenção e cuidado contra a COVID-19 e dezenove vídeos com temas voltados à nutrição e saúde. A interação com os usuários, mesmo que de maneira digital, permitiu a continuidade do convívio social, tão importante para a população em questão, bem como, o repasse de conhecimentos e informações verídicas relacionadas à nutrição e saúde. Contudo, não se destaca a possibilidade da retomada das oficinas presenciais, uma vez que elas permitem uma melhor comunicação e engajamento dos usuários.

Palavras-chave: Atividades educativas; Educação alimentar e nutricional; Saúde mental; Centros comunitários de saúde mental; COVID-19.

\begin{abstract}
With the pandemic context, due to COVID 19, social distancing became essential as a form of prevention, making it necessary for research and extension actions to reinvent themselves. The work in question aims to report the experience of the Nutrition Education for People with Mental Distress project working at the Living and Cultural Center, during 2020, in the context of the COVID-19 pandemic. This is a descriptive experience report. Initially, the approach took place through digital material in the form of flyers sent by the WhatsApp application to users. With the need for greater proximity, animated educational videos were produced, sent to users who were interested in participating in the actions. From March 2020 to March 2021, eight flyers were produced with themes focused on prevention and care against COVID-19 and nineteen videos with themes focused on nutrition and health. The interaction with users, even if digitally, allowed the continuity of social interaction, so important for the population in question, as well as the transfer of knowledge and truthful information. However, the possibility of resuming face-toface workshops is not highlighted, as they allow better communication and user engagement.
\end{abstract}

Keywords: Educational activities; Food and nutrition education; Mental health; Mental health assistance; COVID-19.

\section{Resumen}

Con el contexto de la pandemia, debido al COVID-19, el distanciamiento social se volvió fundamental como forma de prevención, haciendo necesario que las acciones de investigación y extensión se reinventen. El trabajo en cuestión, 
tiene como objetivo reportar la experiencia del proyecto Educación Nutricional para Personas con Sufrimiento Mental en funcionamiento en un Centro de Vida y Cultura en Belo Horizonte-MG, durante el año 2020, en el contexto de la pandemia COVID-19. Este es un informe descriptivo de la experiencia. Inicialmente, el acercamiento se realizó a través de material digital en forma de flyers enviados a través de la aplicación WhatsApp a los usuarios del Centro de Convivência. Con la necesidad de acercarse a los usuarios, se produjeron videos educativos animados, también enviados a través de la aplicación WhatsApp. Desde marzo de 2020 hasta marzo de 2021, se produjeron ocho volantes con temas enfocados en la prevención y atención contra COVID-19 y diecinueve videos con temas enfocados a la nutrición y la salud. La interacción con los usuarios, aunque sea de forma digital, permitió la continuidad de la interacción social, tan importante para la población en cuestión, así como la transferencia de conocimientos e información veraz relacionada con la nutrición y la salud. Sin embargo, no se destaca la posibilidad de reanudar los talleres presenciales, ya que permiten una mejor comunicación y participación de los usuarios.

Palabras clave: Actividades educativas; Educación alimentaria y nutricional; Salud mental; Atención a la salud mental; COVID-19.

\section{Introdução}

A saúde mental no Brasil, até certo momento, foi foco apenas do domínio médico, no qual a exclusão dos indivíduos era um dos principais meios de tratamento. Contudo, nos anos de 1990 ocorreu a Reforma Psiquiátrica que culminou na criação de uma rede de atenção psicossocial com o intuito de dar assistência no cuidado das pessoas em sofrimento psíquico, por meio de uma modelo interdisciplinar em saúde mental, visando a autonomia e inserção desses indivíduos dentro da sociedade (Mattos et al, 2018).

Dentro dessa rede de atenção, os Centros de Convivência e Cultura surgem em 2005, por meio da Portaria $\mathrm{n}^{\circ} 396$, para promover a interação entre os indivíduos, de forma a criar um ambiente sociável e de efetividade. Além disso, tais locais também servem como local de refúgio, de apoio e de aprendizado para muitos daqueles que o frequentam, auxiliando assim no processo terapêutico (Alvarez et al, 2016).

Entre os Centros de Convivência e Cultura existentes no Brasil, em um especificamente localizado em Belo Horizonte - MG, vem sendo realizado um projeto de pesquisa em interface com a extensão cujas atividades consistem em acompanhamento nutricional dos portadores de sofrimento e desenvolvimento de oficinas terapêuticas com temas centrais, relacionados a nutrição, nas quais são realizadas atividades lúdicas, educativas e participativas, de forma que o conhecimento é mútuo entre os participantes. Tais atividades permitem a participação ativa dos presentes, possibilitando expor sentimentos e questionamentos que podem ser debatidos em grupo, de forma a desenvolver a autonomia das decisões e criar um vínculo e interação social (Mattos et al, 2018).

Entretanto, em março de 2020, a Organização Mundial da Saúde (OMS) decretou um contexto pandêmico devido à doença da COVID-19, causado pelo novo coronavírus (Sars-Cov-2), o que culminou na necessidade de distanciamento social como uma das formas de prevenção (OPAS/OMS, 2020; Adhikari et al., 2020; Hoffmann et al., 2020). O impacto de tal medida ainda não pode ser mensurado, principalmente no que diz respeito às possíveis consequências à saúde mental a longo prazo. Dessa maneira, a continuidade das atividades dos projetos de extensão, de maneira segura, é essencial para garantir o vínculo entre universidade e comunidade, principalmente no contexto de vulnerabilidade mental, bem como perpassar informações necessárias e corretas em meio às incertezas e preocupações (Ferrari et al, 2020; Oliveira et al, 2021; Croda et al, 2020).

Assim, devido ao distanciamento social, foi necessário que o projeto em questão se reinventasse e criasse novas abordagens para o processo de educação em saúde, de maneira a continuar a garantir assistência aos usuários do Centro de Convivência. À vista disso, este presente trabalho tem como o objetivo relatar a experiência da reinvenção e continuidade do projeto "Educação Nutricional para Portadores de Sofrimento Mental" durante o ano de 2020, no contexto de pandemia da COVID-19. 


\section{Metodologia}

Trata-se de um relato descritivo de experiência. O relato foi descritivo, de experiência retrospectivo construído a partir da prática vivenciada no projeto Educação Nutricional para Portadores de Sofrimento Mental atuando em um Centro de Convivência e Cultura de Belo Horizonte-MG, durante o ano de 2020, no contexto de pandemia da COVID-19. O relato de experiência, diferentemente dos relatórios científicos, apresenta linguagem mais informal e caráter sintético a fim de proporcionar o enriquecimento da fundamentação teórica com a própria vivência profissional (Santos, 2008; Pereira et al, 2018).

Em 18 março de 2020, a Universidade Federal de Minas Gerais suspendeu todas as suas atividades acadêmicas presenciais, tendo mantido apenas atividades à distância de ensino, extensão e pesquisa. Por conseguinte, os projetos de pesquisa e extensão foram planejados para ocorrerem de forma remota, com ênfase para ações relacionadas à COVID-19.

O projeto para dar continuidade às suas atividades buscou se reinventar por meio da elaboração de materiais lúdicos como os flyers (folders digitais). Inicialmente foram criados elementos em formato de flyers com temáticas baseadas em informações e evidências relacionados à prevenção e cuidado contra a COVID-19. Além disso, também foram elaborados flyers sobre alimentação adequada com objetivo de orientar os usuários do Centro de Convivência dos benefícios para a saúde de uma alimentação baseada em alimentos in natura. Tais temas foram escolhidos devido a importância e a necessidade de informações verídicas a serem repassadas para a população, evitando equívocos e circulação de falsas mensagens.

As temáticas abordadas nos flyers são determinadas pela equipe extensionista juntamente com a gerente do Centro de Convivência de acordo com a percepção das necessidades da população em cada momento da pandemia. Tem-se buscado ampliar a diversidade e a pluralidade de abordagens e de informações, considerando as temáticas relevantes no contexto atual e sobre nutrição e alimentação.

Os flyers são divulgados semanalmente e enviados aos usuários através de aplicativo de rede social, em um grupo criado pela coordenação do Centro de Convivência. O aplicativo de rede social WhatsApp foi escolhido como forma de vincular as informações, devido ser esse o principal meio de envio de mensagens em mais de 120 países do mundo (Fabene et al, 2020). Dessa forma, mais usuários dos outros Centros de Convivência de Belo Horizonte também poderiam ter contato com os materiais criados. Além disso, a plataforma permite o envio não apenas de textos escritos, mas também de imagens, vídeos e áudios de maneira que a compreensão do conteúdo seja a melhor possível.

Com o decorrer da pandemia e a mudança da realidade, observou-se uma demanda por atividades com maior aproximação com os usuários do Centro de Convivência específico do projeto. Dessa forma, foram produzidos vídeos educativos animados e de curta duração com temáticas diversas. Esses vídeos educativos são encaminhados individualmente aos usuários do Centro de Convivência pelo aplicativo WhatsApp, o que permite um diálogo mais interativo, e depois discutidos em formato de oficinas educativas online. As temáticas das oficinas educativas online atendem às demandas dos usuários e permitem a sua participação ativa, possibilitando questionamentos diversos e relacionados à nutrição. Além disso, para reforçar a maior proximidade com os usuários e o momento atual, foram desenvolvidos vídeos temáticos de acordo com o período do ano, como por exemplo, o vídeo temático sobre a alimentação no Natal, dentre outros.

Para esta etapa do projeto de extensão foram definidos aqueles usuários do Centro de Convivência que participavam das oficinas educativas presencialmente, possuíam o aplicativo Whatsapp e tinham interesse em participar das oficinas educativas remotas. Para a elaboração dos flyers e vídeos educativos foram utilizados aplicativos como a plataforma Canva (https://www.canva.com/), a qual permite a estruturação de imagens, textos, cores e elementos; e o programa Shotcut para a montagem e edição dos vídeos, de forma que fosse possível a introdução do áudio dos alunos extensionistas, e de músicas, tornando o material mais dinâmico e atrativo. 
Os materiais educativos produzidos, flyers e vídeos educativos são enviados não somente para o Centro de Convivência no qual as atividades do projeto são desenvolvidas, mas também para todos os Centros de Convivência para Portadores de Sofrimento Mental do município de Belo Horizonte, Minas Gerais. Porém, as oficinas educativas remotas são realizadas somente no Centro de Convivência específico do projeto.

O conteúdo dos flyers e vídeos educativos produzidos é desenvolvido pela equipe extensionista a partir de buscas em artigos, relatórios e publicações oriundas de organizações nacionais e internacionais de saúde, sob orientação da docente coordenadora do projeto.

\title{
3. Resultados e Discussão
}

Ao longo de um ano de pandemia, durante os meses de março de 2020 a março de 2021, elaborou-se como produtos de extensão do projeto "Educação Nutricional para Portadores de Sofrimento Mental" oito flyers com temáticas voltadas à prevenção e cuidados contra a COVID-19, e dezenove vídeos educativos com temas voltados a nutrição e saúde, conforme disposto na Figura 1 e na Tabela 1.

Inicialmente, a equipe do projeto definiu a elaboração dos flyers com o intuito de sensibilizar a população quanto à importância da escolha das informações e de suas fontes, especialmente a respeito do novo coronavírus e sobre a nutrição e saúde no contexto da pandemia da COVID-19.

Figura 1 - Descrição dos títulos dos flyers desenvolvidos entre março de 2020 a março de 2021. Belo Horizonte, 2021.

\author{
Como higienizar as mãos corretamente \\ Como ter uma alimentação adequada durante a quarentena \\ Como higienizar os alimentos em tempos de COVID-19 \\ Como higienizar os objetos e utensílios de sua casa em tempos de COVID-19 \\ Como higienizar as compras em tempos de COVID-19 \\ Cuidados ao comprar alimentos por delivery em tempos de COVID-19 \\ Cuidados na ida ao mercado em tempos de COVID-19 \\ Como fazer horta vertical em casa
}

Fonte: Autores. 
Tabela 1: Descrição das temáticas e títulos dos vídeos desenvolvidos entre março de 2020 a março de 2021. Belo Horizonte, 2021.

\begin{tabular}{ll}
\hline \multicolumn{1}{c}{ Temáticas } & \multicolumn{1}{c}{ Títulos dos Vídeos Educativos } \\
\hline Alimentação e Nutrição & Mitos e verdades sobre a Nutrição \\
& Guia da Alimentar da População Brasileira \\
& Hora de Comer \\
\hline Higienização das Mãos & Como lavar as mãos corretamente \\
\hline \multirow{2}{*}{ Doenças e Alimentação } & Diabetes mellitus \\
& Índice e Carga glicêmica \\
& Uso de medicamentos relacionados à saúde mental e ganho de peso \\
& Sistema Imunológico \\
& Comer compulsivo \\
& Constipação \\
\hline Grupos de Alimentos & Hipertensão \\
\hline Alimentos in natura e Minimamente & Tipos de gordura \\
Processados & O que são carboidratos? \\
& Natal (ceia de Natal) \\
\hline Importância da Prática de Exercícios & Receita de Bolo de Banana \\
Físicos & Receita de Doce de Abóbora \\
\hline Pandemia da Covid-19 & Otivide física e Nutrição \\
\hline
\end{tabular}

Fonte: Autores.

A era da comunicação digital já existia, mas foi impulsionada mediante o distanciamento social, medida de proteção coletiva contra a COVID-19 e tornou-se um paradigma de equidade social. Assim, o conhecimento sobre os canais de comunicação é primordial à garantia universal e equânime ao processo comunicacional em saúde. O avanço do uso de tecnologias da comunicação, incluindo os telefones móveis e mídia digital tem impactado sobre a forma como as pessoas acessam e confiam nas informações de saúde e, assim, gerado desafios de monitoramento e resposta rápida aos conteúdos falsos disseminados via estes meios (Lana et al, 2020).

Conforme relatado na literatura científica e por órgãos oficiais, as notícias e informações falsas podem ter impacto negativo nas pessoas que as recebem, o que pode contribuir para agravar o curso da pandemia da Covid-19 (VasconcellosSilva \& Castiel, 2020). Nesse contexto, torna-se importante reconhecer notícias falsas a fim de prevenir a desinformação, visto que a disseminação desses conteúdos contribui para o descrédito da ciência e instituições globais de saúde (Galhardi et al, 2020). Evidencia-se, ainda, a importância da elaboração de conteúdos que levem em consideração a clareza e as evidências científicas, pois a educação em saúde é uma estratégia eficiente para combater a desinformação ajudando assim a salvar vidas (Tangcharoensathien et al, 2020).

A educação em saúde, desde muito, vem sendo uma aliada na prevenção e promoção à saúde. Neste momento de incertezas e preocupações, as ações do projeto de extensão com a propagação de informações baseadas em evidências científicas, surge como um auxílio no combate ao coronavírus (Pereira \& Palma, 2018). Além disto, o cuidado adequado em saúde mental é um tema que vem sendo discutido no Brasil desde o início da Reforma Psiquiátrica, sendo priorizada uma assistência integral com enfoque não apenas aos indivíduos em sofrimento mental e às suas famílias, mas também no desenvolvimento de ações de promoção e prevenção aos agravos de saúde mental na população (Barros et al, 2019).

A escolha da temática de cada um dos flyers respeitou a identificação das necessidades dos usuários do Centro de Convivência em cada momento da pandemia, bem como da necessidade observada. Assim, a escolha das temáticas dos flyers ocorreu ao reconhecer que, durante a pandemia, a rotina da população precisou ser modificada para a nova realidade (Bezerra 
\& Palma, 2018). Dessa maneira, o desenvolvimento dos flyers teve o intuito de fornecer informações em relação aos cuidados higiênico-sanitários à população alvo para o enfrentamento da pandemia.

Dessa forma, foram definidas temáticas voltadas para a higienização de mãos, alimentos, objetos, compras, bem como, a maneira como se portar em comércios, com o objetivo de auxiliar a população-alvo a se proteger (Oliveira et al., 2021) (Figuras 2, 3, 4, 5).

Figura 2 - Como higienizar as mãos corretamente em tempos de Covid-19.

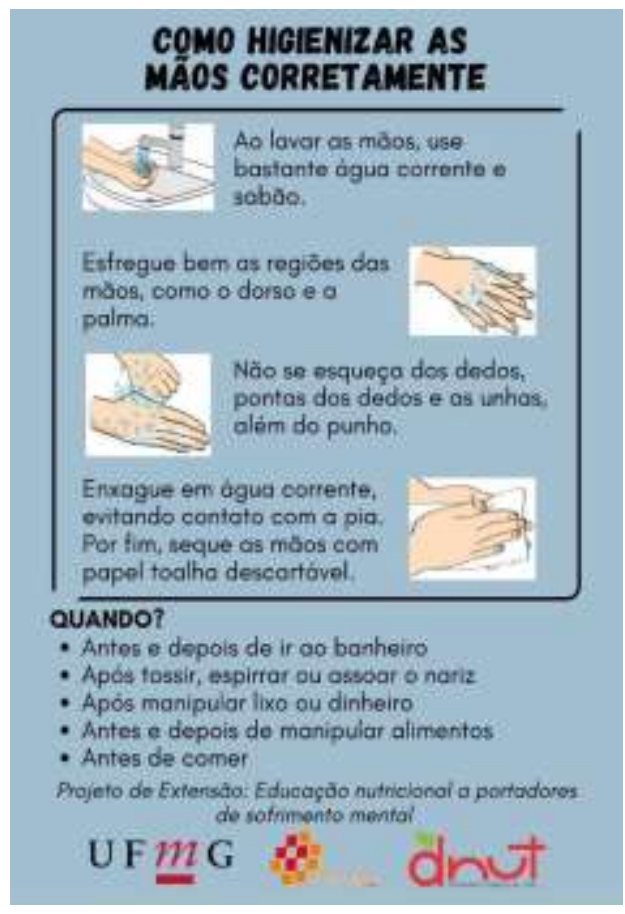

Fonte: Autores (2020).
Figura 3 - Como higienizar as compras em tempos de Covid-19.

\section{COMO HIGIENIZAR AS COMPRAS EM} TEMPOS DE COVID-19

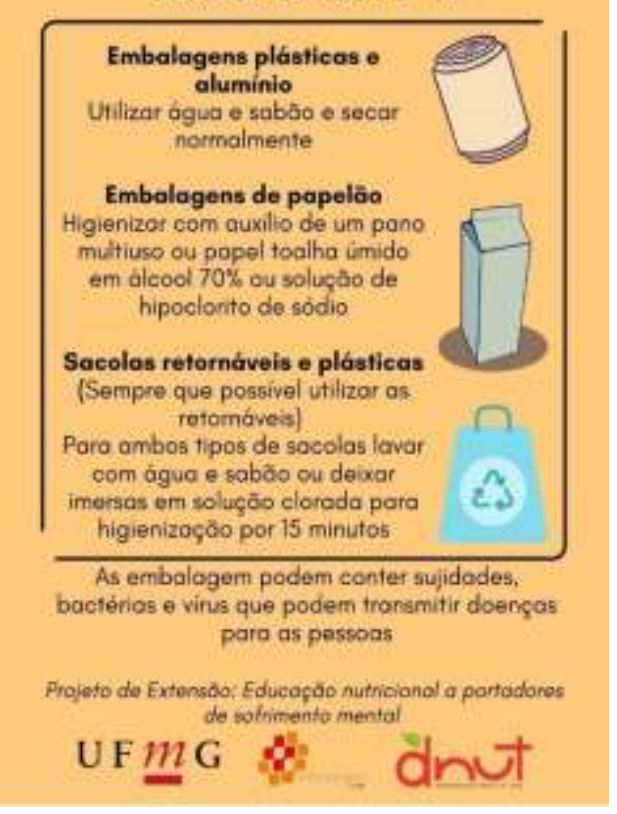

Fonte: Autores (2020). 
Figura 4 - Como higienizar os objetos e utensílios de sua casa em tempos de Covid-19.

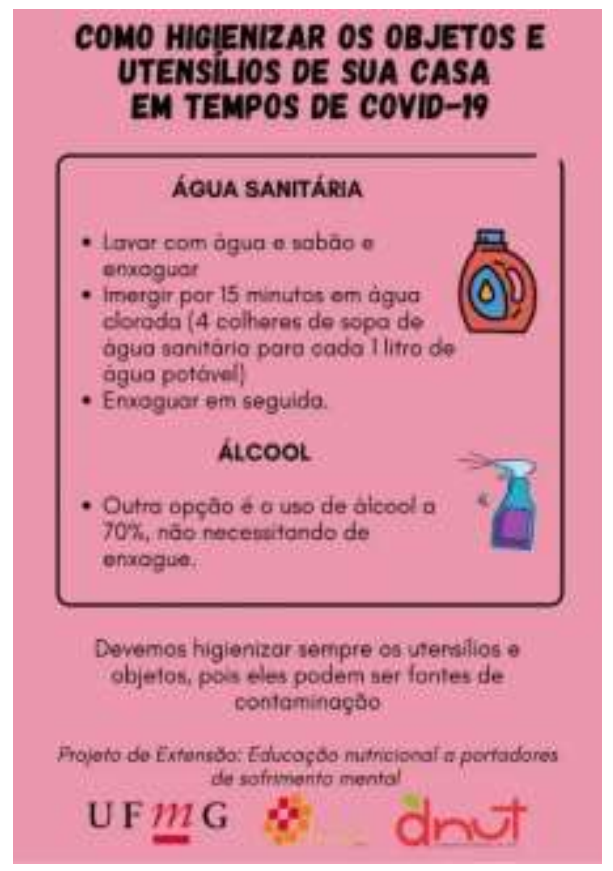

Fonte: Autores (2020).
Figura 5 - Como higienizar os alimentos em tempos de Covid-19.

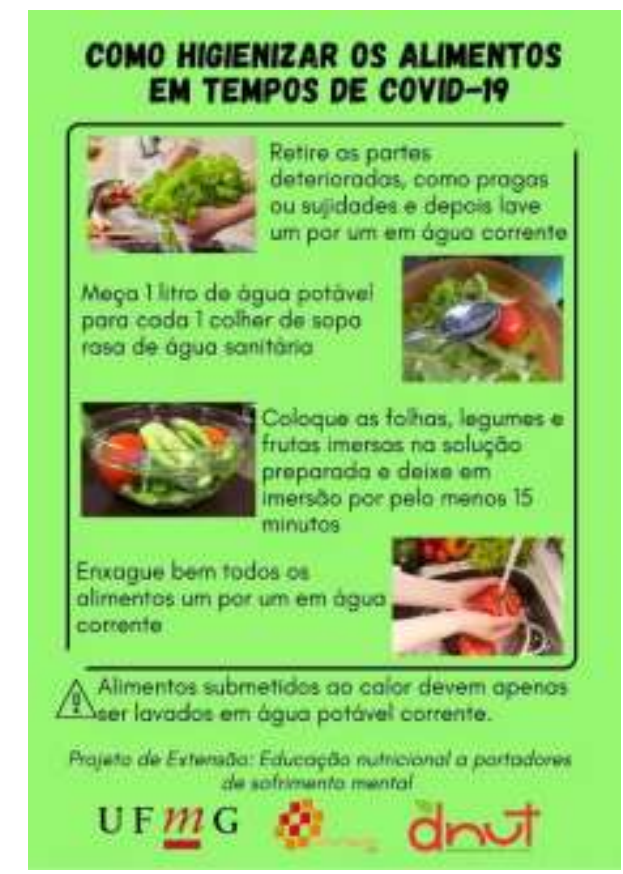

Fonte: Autores (2020).

O último flyer, "Como fazer horta vertical”, foi uma iniciativa de tentar amenizar a insegurança alimentar que muitas famílias estão enfrentando na pandemia devido ao desemprego e à inflação dos alimentos. Além disso, o cultivo de plantas serve como uma atividade terapêutica, ajudando no controle da ansiedade, depressão e outros sofrimentos mentais ${ }^{1}$.

O feedback dos usuários dos Centros de Convivência em relação aos flyers foi muito bom, ressaltando que os flyers foram enviados não apenas para os participantes do Centro de Convivência do projeto, mas para todos os usuários dos Centros de Convivência de Belo Horizonte. Por esse motivo não foi possível mensurar quantitativamente o alcance e impacto que esses informativos tiveram na vida dos usuários dos Centros de Convivência.

Com o passar do tempo, a utilização da rede social como WhatsApp se tornou a única forma de contato com os usuários, sendo essencial este vínculo virtual para a continuidade da aproximação e inserção social dos usuários, ainda mais neste momento de distanciamento social (Pereira; Palma, 2020). Dessa maneira, foi definido em reunião pela equipe do projeto, juntamente com os profissionais do Centro de Convivência, alterar a forma como as oficinas seriam realizadas.

Para ser algo ainda mais atrativo e dinâmico foi definido a criação de vídeos educativos com temáticas voltadas à nutrição, a serem definidas de acordo com a equipe do projeto e a demanda dos usuários (Figura 2). Outra mudança que ocorreu nesta etapa do projeto de extensão foi iniciar o contato direto entre os extensionistas e os usuários, pelo WhatsApp, de maneira individual. Essa interação mais próxima, entre acadêmicos e sociedade, mesmo que online, permite um compartilhamento de saberes importantes para a formação de profissionais mais capacitados e humanizados com a realidade brasileira (Ferrari et al, 2020).

A estratégia de definir e dirigir mensagens para um público-alvo garante que os destinatários identifiquem os elementos e o contexto da comunicação, e se tornem, mesmo que minimamente, emocionalmente envolvidos, tendendo a aceitar e reproduzir os comportamentos orientados na campanha (Fabene et al, 2020). Como o objetivo principal desse projeto 
de extensão não é somente transmitir a informação, mas, também, aumentar as chances de que os usuários reproduzam e repassem os conhecimentos obtidos, essas mudanças de formato se deram após observação e avaliação da interação dos usuários de acordo com as estratégias utilizadas.

$\mathrm{Na}$ criação dos vídeos educativo optou-se que em algum momento o rosto dos extensionistas aparecesse, com o objetivo de tornar as oficinas mais intimistas e humanizadas apesar do distanciamento social. Com este vínculo direto estabelecido entre os extensionistas do projeto e os usuários do Centro de Convivência, tornou-se possível uma maior compreensão das demandas dos usuários, e essa proximidade gerada possibilitou o envio de dúvidas e retorno rápido das respostas, facilitando a compreensão dos extensionistas do seu público alvo, e definição de melhor forma de abordagem dos temas e linguagem.

Apesar do WhatsApp ser uma excelente ferramenta de comunicação neste momento de distanciamento social, ainda há algumas situações que dificultam o desenvolvimento das oficinas educativas com um maior número de usuários, como a pouca afinidade com a tecnologia digital que alguns indivíduos têm ou até mesmo não possuírem o aplicativo ou um smartphone (Fabene et al., 2020).

Ao longo desse período em que o projeto está no formato remoto a interação entre a equipe extensionista e os participantes das oficinas educativas foi se adequando a realidade, sendo que em algumas semanas os usuários respondem com dúvidas, elogios e/ou sugestões, e em outras semanas apenas visualizam o vídeo sem nenhum tipo de comentário. Quando os participantes das oficinas educativas interagem, as respostas costumam ser comentários sobre alguma recomendação citada no vídeo, ou algumas dúvidas relacionadas especificamente com a sua vivência ou de algum familiar próximo. Quando são respostas rápidas costumam ser elogios como "interessante", "bacana", "muito legal" ou algum tipo de emoji que confirme o recebimento.

Quando questionados sobre a continuidade das oficinas educativas no formato remoto e a sua opinião sobre as mesmas, todos apresentam interesse em continuar participando das oficinas, e relatam que os vídeos são muito interessantes. Além disso, justificam o não retorno uma vez ou outra às publicações devido à falta de tempo. Além da falta de tempo citada, devemos considerar também que devido ao público-alvo do projeto ser portador de sofrimentos mentais, é compreensível que uma vez ou outra o usuário possa vir a ter um dia ou uma semana ruim, o que justifica a ausência de respostas.

A pandemia da Covid-19 possibilitou o fortalecimento da era da tecnologia, elencando o seu uso por parte dos cuidadores em saúde mental como uma estratégia para manter o vínculo terapêutico e a continuidade do tratamento (Lopes et al, 2021).

Compreende-se que ações governamentais são essenciais para o enfrentamento dos efeitos sociais e econômicos da pandemia, bem como para a prevenção do surgimento de outras doenças e agravos. Contudo, as ações da sociedade civil e de organizações sociais no exercício de ações de solidariedade e suporte social também são necessárias. Reconhece-se que quando as pessoas se dispõem a ajudar o próximo o senso de responsabilidade social é fortalecido, além de obter benefícios pessoais, pois ajudar outras pessoas pode contribuir para tirar o foco dos próprios problemas, sendo uma estratégia de promoção à saúde mental (Oliveira et al, 2021).

$\mathrm{O}$ apoio durante a pandemia tem sido fundamental para a população vivenciar as restrições sociais e as regras sanitárias necessárias para o convívio social, observa-se grande mobilização em diferentes países, quer seja de forma direta, como o uso de máscara e o respeito ao distanciamento social, ou indireta, em especial por meio das redes sociais, exemplificado como mensagens de apoio, motivação e orientações. Diferentes estratégias, entre elas o voluntariado, as políticas públicas de alguns países em prol da proteção de seus cidadãos foram utilizadas nesse sentido, uma rede de solidariedade se formou e a resiliência social tem sido importante na vida das pessoas (Carlsen, Toubol \& Brincker, 2021). Consequentemente, a pandemia tem permitido pensar no próximo com mais humanização e responsabilidade (Oliveira et al., 
2021). Além disto, reorganizar e reinventar o cuidado são atitudes que fornecem ao usuário da assistência mental significado social e humanitário, mesmo diante de momentos de crise (Lopes et al, 2021).

Entre as limitações desse projeto neste momento, cita-se a realização das atividades de forma remota, o que pode não atingir todos os usuários do Centro de Convivência, considerando a desigualdade digital existente na sociedade brasileira. Uma segunda limitação está relacionada à ausência de uma avaliação completa da ação educativa, em virtude do material educativo está sendo divulgado a todos Centro de Convivência, e dessa forma não é possível mensurar o seu impacto na qualidade de vida de todos os portadores de sofrimento mental que têm acesso a esse material.

\section{Considerações Finais}

Apesar das dificuldades encontradas ao longo da realização do projeto remotamente, é possível observar a importância de dar continuidade nas oficinas educativas. Neste momento em que vivemos de isolamento social devido a COVID-19, ações que aproximem as pessoas mesmo que minimamente torna-se de extrema importância para uma melhor saúde mental. Além do projeto colaborar para isso, ele também estimula os participantes a manterem uma vida mais saudável mesmo diante do cenário atual, cuidando de sua alimentação e incentivando a prática de atividade física.

Mesmo que as oficinas remotas sejam uma possibilidade de continuidade das atividades do projeto de extensão nesse momento de pandemia, não descartamos a possibilidade e importância de a posteriori retornarmos com as oficinas educativas presenciais, principalmente por permitirem uma melhor comunicação com os usuários e um maior controle de participação dos usuários em cada oficina.

\section{Referências}

Alvarez, A. P. E., da Silva, J. O., \& de Moraes Oliveira, A. C. (2016). Centro de Convivência e Cultura: diálogos sobre autonomia e convivência. ECOSEstudos Contemporâneos da Subjetividade, 6(1), 5-19. http://www.periodicoshumanas.uff.br/ecos/article/view/1859

Adhikari, S. P., Meng, S., Wu, Y. J., Mao, Y. P., Ye, R. X., Wang, Q. Z., \& Zhou, H. (2020). Epidemiology, causes, clinical manifestation and diagnosis, prevention and control of coronavirus disease (COVID-19) during the early outbreak period: A scoping review. Infectious Diseases of Poverty, 9(29), 1-12. https://doi.org/10.1186/s40249-020-00646-x

Barros, S., Nóbrega, S. P. S M., Santos, C. J., Fonseca, M. L., \& Floriano, M. S. (2019). Saúde mental na atenção primária: processo saúde-doença, segundo profissionais de saúde. Revista Brasileira de Enfermagem, 72(6), 1687-95. https://doi.org/10.1590/0034-7167-2018-0743

Bezerra, A. C. V., Silva, C. E. M. D., Soares, F. R. G. \& Silva, J. A. M. D. (2020). Fatores associados ao comportamento da população durante o isolamento social na pandemia de COVID-19. Ciência e Saúde Coletiva, 25, 2411-2421. https://doi.org/10.1590/1413-81232020256.1.10792020

Carlsen, H. B., Toubol, J. \& Brincker B.(2021). On solidarity and volunteering during the COVID-19 crisis in Denmark: the impact of social networks and social media groups on the distribution of support. European Societies, 23(sup1), S122-S140. https://doi.org/10.1080/14616696.2020.1818270

Croda, J., Oliveira, W.K., Frutuoso, R.L., Mandetta, L.H., Baia-Da-Silva, D.C., Brito-Sousa, J.D., Lacerda, M.V.G. (2020). COVID-19 no Brasil: vantagens de um sistema único de saúde socializado e preparação para contenção de casos. Revista da Sociedade Brasileira de Medicina Tropical, 53 , e20200167. https://doi.org/10.1590/0037-8682-0167-2020

Fabene, M. R., Voltareli L. C., de Andrade, G.R., Lucena, T. F. R. \& Yamaguchi, M. U. (2020). Ação de Comunicação em saúde no WhatsApp com base no perfil digital de portadores de hipertensão e diabetes. O Mundo da Saúde, 1(44): 12-22. https://doi.org/10.15343/0104-7809.201944012022

Ferrari, J. M. L., Bartholo, B. R., Santana, M. I. T. \& Coelho, H. C. (2020). Desafios e possibilidades da extensão universitária diante do COVID-19: análise do projeto "Construindo Lugares de Urbanidade Metropolitana". Revista Científica Foz, 3(1), 24-24. https://revista.ivc.br/index.php/revistafoz/issue/view/8

Hoffmann, M., Kleine-Weber, H., Krueger, N., Mueller, M. A., Drosten, C. \& Poehlmann, S. (2020). The novel coronavirus 2019 (2019-nCoV) uses the SARS-coronavirus receptor ACE2 and the cellular protease TMPRSS2 for entry into target cells. BioRxiv, 18, 271-280. https://doi.org/10.1016 / j.cell.2020.02.052

Galhardi, C. P., Freire, N. P., Minayo, M. C. D. S. \& Fagundes, M. C. M. (2020). Fato ou Fake? Uma análise da desinformação frente à pandemia da Covid19 no Brasil. Ciência \& Saúde Coletiva, 25:4201-4210. https://doi.org/10.1590/1413-812320202510.2.28922020

Lana, R., Coelho, F., Gomes, M., Cruz, O., Bastos, L., Villela, D. \& Codeço, C. (2020). Emergência do novo coronavírus (SARS - CoV - 2) e o papel de uma vigilância nacional em saúde oportuna e efetiva. Cadernos de Saúde Pública, 36(3), e00019620. https://doi.org/10.1590/0102-311X00019620 
Research, Society and Development, v. 10, n. 15, e139101522858, 2021

(CC BY 4.0) | ISSN 2525-3409 | DOI: http://dx.doi.org/10.33448/rsd-v10i15.22858

Lopes, L., Jorge, M. S. B., Silva, D. M. F. da, Oliveira, R. S., Barroso, P. \& Lourinho, L. (2021). O cuidado em saúde mental no centro de atenção psicossocial (caps) em tempos de Covid-19: revisão integrativa. Research, Society and Development, 10(11), e174101119516. https://doi.org/10.33448/rsd-v10i11.19516

Mattos, A. C. E, da Silva, Rocha L. \& Rodrigues, L. (2018). Dialogando sobre alimentação e nutrição na saúde mental: Ações promotoras de saúde por meio de oficinas de horticultura. Revista da Associação Brasileira de Nutrição-RASBRAN, 9(2), 17-24. https://www.rasbran.com.br/rasbran/issue/view/18

Oliveira, R. M. de, Prates, M. L. S., Prates, E. J. S., Santos, T. C. C. \& Reinaldo, A. M. S. (2021). Saúde mental e a COVID-19: intervenções virtuais de educação em saúde para o enfrentamento da pandemia. Expressa $\quad$ Extensão, 26(1), 675-696. https://periodicos.ufpel.edu.br/ojs2/index.php/expressaextensao/article/view/19686

OPAS/OMS Brasil. (2020). Folha informativa COVID-19 (doença causada pelo novo coronavírus). Brasília: Organização Pan-Americana de Saúde. https://www.paho.org/bra/index.php?option=com_content \&view=artcle\&id=6101:covid19\&Itemid=875

Pereira, O. P. \& Palma, A. C. (2018). Sentidos de consultórios terapêuticos ocupacionais de CAPS não cotidianos de dois usuários: uma descrição fenomenológica. Revista de Abordagem Gestáltica: Estudos Fenomenológicos, 24 (1), 15-23. http://dx.doi.org/10.18065/RAG.2020v26n1.10

Pereira A. S., Shitsuka, D. M., Parreira, F. J., \& Shitsuka, R. (2018). Metodologia da pesquisa científica. [e-book]. (Ed.1). Santa Maria, RS: UAB/NTE/UFSM. https://repositorio.ufsm.br/bitstream/handle/1/15824/Lic_Computacao_Metodologia-Pesquisa-Cientifica.pdf?sequence=1

Santos, A. R. (2008). Metodologia científica: a construção do conhecimento. Rio de Janeiro: Lamparina.

Tangcharoensathien, V., Calleja, N., Nguyen, T., Purnat, T., D’Agostino, M., Garcia-Saiso, S, \& Briand, S. (2020). Framework for managing the COVID-19 infodemic: methods and results of an online, crowdsourced WHO technical consultation. Journal Medical Internet Research, 22(6), e19659 https://doi.org/10.2196/ 19659

Vasconcellos-Silva, P. R. \& Castiel L. D. COVID-19, fake news, and the sleep of communicative reason producing monsters: the narrative of risks and the risks of narratives (2020). Cadernos de Saúde Pública, 36(7), e00101920. https://doi.org/10.1590/0102-311X00101920 